by a Roman 2, whilst its abnormal state is indicated by the same figure in italics. The relative positions of the four different anatomical facts is retained throughout :TABLE I.

\begin{tabular}{|c|c|c|c|c|c|c|}
\hline & Carotids. & Ambiens. & Furcula. & Oil-gland. \\
\hline (I) & PALEORNITHIN & & 2 & - & + & + \\
\hline (2) & STRINGOPINÆ & - & 2 & $\vec{\imath}$ & - & + \\
\hline (3) & ARINE & - & 2 & + & + & + \\
\hline (4) & PYRRHURINAE & . & 2 & - & + & + \\
\hline (5) & PLATYCERINA & - & 2 & - & - & + \\
\hline (6) & CHRYSOTIN & - & 2 & 一 & + & - \\
\hline
\end{tabular}

On this arrangement, the Lories, belonging to the Palceornithina, their zoological formula is $2-++$; whilst that of Cyanorhamphus, which is one of the Platycercince, is $2--+$ By this means the relations of the different groups to one another are readily recognisable.

Next, in the attempt to arrive at a correct detailed classification, the question as to the zoological formula of the ancestral Psittacine form must be one of primary importance. This can only be arrived at by a comparison of the other bird-types with that of the parrots. Taking the characters employed in Table I., and similarly formulating such birds as the fowl, duck, rail, stork, and cuckoo, they all agree in being represented by $2+++(\mathrm{I})$; others, like the kingfishers and hornbills, have the formula $2-++(2)$; whilst a third type, with only a left carotid, are included in the $\mathrm{L}-++$ type (3). No others of importance exist. From which of them did that of the Psittaci spring? It must have been from one; and, peculiarly enough, there are genera to be found among them which closely approach all three, for-m

$$
\begin{gathered}
\text { The formula of Pittacus is } 2+++ \\
\text { " "Palcornis " } 2-++
\end{gathered}
$$

However, this only shows that the sub-order is a very ancient one, and has undergone changes analogous to the whole class Aves, and it does not complicate the problem in the least.

There are parrots with two normal carotids, e.g. the Palcornithince; there are others in which the ambiens is present, e.g. the Arince; most have a furcula and also an oil-gland.

Now suppose that when steam-engines were first introduced they had all been constructed with steam-whistles attached. Suppose that shortly afterwards several had been exported to different colonies, and that ever afterwards each colony had, with the originals as patterns, gone on constructing them for their own use, improving upon the original design as they thought best. Suppose that by certain individual manufacturers a gong was substituted for the whistle; in others a bell, and in a third no sounding apparatus at all. A traveller going through the different countries at the present time would probably find whistle-engines wherever he went, though in different places gongs or bells will have replaced the whistle. Knowing nothing about the history of the steam-engine, is he not justified in inferring that it was originally constructed with a whistle; for otherwise would it be likely that cach colony should have independently employed the same method of signalling, when there were several to be chosen from?

The naturalist, similarly, as an uninitiated looker-on at the contrivances of nature, finds the same type of structure running through forms not very intimately allied; as, for example, two symmetrical carotids, in reptiles, mammals, and some birds; or an ambiens muscle in the fowl, the eagle, the cuckoo, and the plantain-cutter. When, therefore, these fundamental arrangements are found to exist (though perhaps not combined in any one individual) in any well-defined group like the parrots, may it not be legitimately inferred that the ancestor of that group possessed them in their full and unmodified form? Undoubtedly it may; and on this principle we can almost certainly assume that the ancestral parrot possessed two normal carotids, an ambiens muscle, a complete furcula, and an oil-gland; in fact, that its formula was $2+++$; and that all those species in which one or other of the included characters differ from this type formula, they do so on account of forces having modified the ancestral form. This line of argument therefore leads us to infer the extinction of the earliest form of parrot, unless some yet undissected genus is subsequently found to correspond with it ; and all the existing genera must be referred to collateral branches, in which at least one operation of modification has been accomplished. Those which have undergone no further change from the $2+++$ type are the Palaornithina $(2-++)$, and the Arina $(2+++)$ Now the question presents itself, are all those with the modified carotid (2), members of a single stem, and those with the unmodified carotid (2) members of another, similar losses having occurred in both to develop the subjoined series?

$$
\begin{array}{l|l}
2-++ & 2++t \\
2-+ \pm & 2-++ \\
2-+- & 2-1+ \\
& 2-+-
\end{array}
$$

Or must those types be blended in which the formulæe correspond, irrespective of the carotids? My placing the carotid index first expresses my belief as to its primary importance ; and this is because the conformation it represents is extremely peculiar and unique among birds, and is therefore less likely to have appeared except as the operation of a specially applied force on a single collection of individuals, the power of transmission being inherited. From this it may be inferred that the ancestral unmodified stem shortly sent off a branch represented br $2++t$, which persists as such in the Arince. The main stem and its branch must each have, before long, had a branch of its own, represented by $2-++$ and $2-++$, which persist as the Palceornithince and the Pyrrhurince. From the $2-++$ division sprang the $2-\ldots+$ (Stringopince), and the genus Cacatua $(\mathrm{L}-+-)$, as did the $2--+$ (Platycercince) and the $2-+-($ Chrysotina $)$ from the $2-++$ division. The genus Cacatua is peculiar in having only the left carotid running normally, it must therefore be connected with the normal 2 carotid stem, and many Cacatinnæ, like the Cockateel and the Banksian Cockatoo, are represented by the formula $2-++$. Some of the true Cockatoos, and some only, have no oil-gland.

My object in giving this somewhat lengthy illustration on the present occasion is to show how much facility a method of formulation affords in the working out of a minor problem of the great doctrine of heredity, such as the classification of the parrots. It makes comparison easy, it facilitates the performance of operations of addition and subtraction, bringing all the stages of the process before the mind's eye without any mental effort. Is it not one to be further developed?

\section{THE OPTICS OF THE SPECTROSCOPE}

$\mathrm{NOW}$ that the Spectroscope is becoming an instrument of world-wide use, we think it will be not uninteresting to call attention to some few points that appear to be often overlooked in designing the instrument for various purposes; and in order to ascertain the best arrangement, we cannot do better than analyse the effects produced in any spectroscope by varying the proportion of its parts. We must, however, premise by saying that the power of an instrument is not altogether dependent on the dispersive power of the prisms, but also on the width of the imzge of the slit in the eyepiece of the tele. 
scope of the spectroscope. To make our meaning clear, let us suppose that the slit is illuminated with a sodium flame, then the dispersive power of the prisms will produce in front of the eyepiece two images, or "lines," and with the same lenses the distance of their centres will depend upon the prismatic power; but it is clear that if the slit be widened, the two images will eventually widen until they touch each other or overlap. There is, then, the same dispersion, but less separation, than when we use the narrow slit ; and it would follow from this that with an almost indefinitely small slit a prism of very small dispersion would give two separate images of a sodiumilluminated slit, which could be magnified so as to have their distance and width the same as would be given by using a wider slit and greater prismatic dispersion; but with an eyepiece of the large power required, the lines would be so diminished in brightness as to preclude this arrangement ; and in order to see a spectrum as brilliant as possible, the eyepiece ought to be as low in power as possible consistent with reducing the cylinder of rays sufficiently small that they all enter the lens of the eye.

Let us now consider a spectroscope of any number of prisms having the focal length of the collimator the same as that of the telescope : then the image of the slit in the focus of the telescope will be of the same size and of the same brightness; for we must, for this consideration, omit the loss of light by reflection and absorption for the present, as the slit itself, which we will first suppose illuminated by sodium light, so that two yellow images of it will be visible in the eyepiece. Afterwards we will consider the case in which sun-light is used. First let us consider the effect of opening the slit wider, say double the width. By this means the images will be doubled in width and the separation diminished; the amount of light will be doubled, but will be spread over double the area, so the intensity of illumination will remain the same; therefore the slit should be as narrow as possible consistent with the image being wide enough to be visible. Secondly, let us double the length of the collimator. By this we halve the width of the image of the slit, so that the separation is increased, but the distance between the centres of the lines remains the same; the angle subtended by the collimating lens will in this case be halved, so that the amount of light passing will only be $\frac{1}{4}$ of the original amount, but as the image of the slit is reduced in like proportion, the intensity of illumination remains the same; the effect in this case is therefore the same as narrowing the slit, with the exception of the lines being shorter, thereby reducing the width of the spectrum-a matter generally of little moment, which can be altered at ease by lengthening the slit, Thirdly, we will double the diameter of the collimating lens, and with it that of the telescope and the prism. By these alterations the amount of light passing becomes quadrupled, therefore the images of the slit will be four times brighter; but the angle subtended by the telescope lens at the image is doubled, so that in order to get the whole of the light into the eye, the eyepiece must be placed at half its distance from the image, and be consequently doubled in power; the images will by this be reduced to their original brightness, but they will be magnified at the same time, and the distance from centre to centre doubled, the separation doubled, and the width of the images doubled, so that the slit may be reduced in width by $\frac{1}{2}$, and yet leave each image as wide as at first. This will increase the separation between the interior sides of the image still more, so that by doubling the size of our lenses and prisms we have obtained double separation of centres of images, and more than double separation between images, which is just what would be produced by doubling the number or dispersive power of the prisms. It is therefore obvious that in dealing with a bright-line spectrum the power of the instrument depends on the size of the prisms as much as on their number, and an increase in number means an increase in the number of reflecting surfaces and loss of light, so that within practical limits an increase of size is the more preferable. Practically, on increasing the size of the collimating lens, as in this case the focal length should be increased, otherwise the lens is injured in defining power, the effect of this increase is, as shown in the second case, only equivalent to closing the slit, so it is better to lengthen the collimator instead of touching the slit; it is also better to increase the focal length of the telescope glass, thereby straining it less, and so increasing the size of the image of the slit without altering the power of the eyepiece.

Now let us consider the effect of these alterations on sun-light or other light giving a dark-line spectrum; and there is this difference between the consideration of this spectrum and the bright-line spectrum, for in this case the dark lines are not images of the slit, but intervals between them, and therefore their width and appearance depend not so much on the separation between the centres of the bright lines as on the separation of their adjacent sides, and with the same width of any two bright lines this separation or width of dark line does not vary in the same ratio as the distance between the centres of the bright lines, or as what is called the dispersive power, varies, but in a higher ratio. For example: suppose there appear in a spectroscope the two sodium lines of appreciable width with the finest possible dark line between them; then, if the distance of their centres is cloubled without increasing their width, the black line becomes increased by the increment of the distance of their centres, and with this increment the original dark line becomes much more than doubled; this will be seen better by drawing two bright lines of appreciable width on paper, and going through the process just mentioned. It is therefore separation, according to our definition of the word, that is required for dark-line spectra.

We will now consider the effect when using sun-light instead of sodium light in a similar manner to our first arrangement, namely, in our normal spectroscope, and let us widen the slit as we did before. Every image of the slit will then widen, and the separation between the sides of any two images will diminish, and therefore the dark lines will diminish in width as they are encroached on by the light on either side; the general spectrum will, however, increase in brilliancy, for although each image is only increased in size, as was the case with sodium light, still the images of each colour overlap, and so produce greater intensity. From this we gather that to obtain the greatest number and width of dark lines, the width should be as narrow as is compatible with sufficient illumination of the spectrum, to show up the dark lines; and so with a dark-line spectrum as with a bright-line one, the slit should be as narrow as possible.

Secondly, as with the sodium light, let us lengthen the collimator, say double it: then, as with the sodium light, the images will be halved and the separation increased, but only $\frac{1}{4}$ of the light passes, and the spectrum is reduced in width by $\frac{1}{2}$, so that its brilliancy is $\frac{1}{2}$ what it was originally; or we may account for the decrease in briliancy by considering that although, as we showed in the case of the sodium light, the images of the slit are not reduced in brilliancy, still there is less overlapping and so less brilliancy. So we see that in order to keep a sufficient brightness of spectrum to show the dark lines, we must open our slit if we lengthen our collimator, and vice versâ, so that no power is gained by either of these methods, as was the case with the sodium light. Thirdly, we will double the diameter of the collimating lens, and with it that of the prisms and telescope object-glass. By this means the brilliancy only of the spectrum is changed, and this is quadrupled in the focus of the eyepiece, but the focal length must be halved in order to reduce the cylinder of rays small enough to totally enter the eye: this willimagnify the spectrum to double its original size in every direc- 
tion, and so double the width of the dark lines, but will produce no new ones; it will also reduce the brightness of the spectrum to its original state. Now, when we were dealing with sodium light, we at this stage of proceeding halved the width of the slit, for the images of the slit had been doubled without their brightness being reduced, so we could halve them and bring them to their original size, and so increase the distance of separation still more; but with a continuous spectrum, if we close the slit we shall, it is true, only decrease the width of each image of the slit and not their brightness, but we decrease their overlapping and so decrease the brilliancy of the whole spectrum, and this we cannot afford to do, as we have started with as narrow a slit as possible, and consequently with as small a brilliancy as possible consistent with showing the dark lines. We have therefore by this alteration of size of glasses doubled the width of dark lines originally visible, but we are not able to more than double the separation of any two images of the slit, as we did with the sodium light images, by narrowing the slit in addition to increasing the distance of the centres, and therefore no new lines are produced; in fact, the result of our change of arrangement has been the same as a simple magnification of the spectrum without a decrease in brilliancy; and an increase of prismatic power is exactly similar in effect, as we shall presently show, though it seems at first untrue that increase of prismatic power will not increase the number of dark lines visible. Let us now double the number of prisms; then the length of the spectrum will be doubled, and the distance of the centre of the images of the slit doubled, and therefore more dark lines may appear in addition to the original ones being widened, but the brilliancy of the spectrum has been halved, and in order to brighten the spectrum to the original state the width of the siit must be doubled, which exactly undoes all that the extra prisms have done in producing more lines; for the images will expand and obliterate the newlyformed lines; the original dark lines will, however, after the widening of the slit, be double their original width; so that, as we have just stated, the increase of prismatic power will not make a greater number of dark lines visible. If we illuminate the slit more intensely, we may decrease the width of the slit and still retain our original brightness, and so obtain a reduction in the width of the images, and consequently a greater separation between their edges, and therefore an increase in the number of dark lines in addition to increase of width of those originally visible; so that for the same kind of light the number of dark lines depends on the intensity of the illumination of the slit.

In dealing with the spectrum of an intense light like that of the sun, where there are a large number of lines, it is necessary to use an instrument of high power, whether in number or size of prisms, in order that the exceedingly fine dark lines produced by a low power may be, as it were, magnified without loss of light, which is, as we have shown, the effect of an increase of prismatic power; and in order that these fine lines may become visible and sufficiently separated to render their identity for measurement or otherwise complete, so there may be an apparent increase in the number of lines by the invisible ones being rendered visible by magnification without loss of brilliancy in the spectrum.

But in dealing with light like that from a planet or the moon, where the slit must be so wide that few lines are visible, it can soon be tested in practice that the increase of power does not increase the number of lines. In examining the light of the moon or of a nebula, or any object having an appreciable diameter, any increase of telescopic power for the purpose of forming the image on the slit will not increase the useful brightness of the slit; for, supposing a spectroscope be working to its greatest advantage on a telescope, then, if the diameter of the object-glass of the telescope be doubled, the angle it subtends at the slit will be doubled, and the cone of rays on the collimator side of the slit will have its base doubled, and therefore it cannot all pass through the collimating lens; in fact, all the rays newly added by the increase of diameter of object-glass will be wasted against the tube of the collimator, and if we try to utilise these rays by increasing the size of collimating lens or decreasing its focal length, we shall also have to increase the power of the eyepiece to get all the rays into the eye, and so reduce the brilliancy of the spectrum to its original state. In the case of increasing the focal length of a telescope as well as its aperture, the brightness of the image on the slit is not increased, but only its size; so the spectroscope is unaffected. But in the case of viewing the spectrum of a star, matters are altered, for the image of the star does not increase in size by increasing the focal length of the telescope together with its apertures; but its brilliancy is increased, and therefore greater prismatic power can be used without increase of width of slit, and more dark lines seen; so that for stellar spectroscopy an increase of telescopic apertures is a direct advantage. From the foregoing remariss we gain that in the construction of a spectroscope the eyepiece should be of as long a focus as possible, so as just to cause all the rays to enter the eye; all magnification beyond this means loss of brilliancy, and if the spectrum appea is insufficiently large an increase in size of the collimatin $\mathrm{g}$ and telescope lenses, together with the prisms, or an increase in the number of the prisms should bo nade, until the spectrum appears sufficiently larse to suit the requirements of the observer.

G. II. S.

\section{THE SUB-WEALDEN EXFLORATLON}

THE Secretary of the Sub-Wealden Exploration has just issued his eighth quarterly report, in which he states that but little progress has been made during the last three months in consequence of the inability to procure lining tubes of the required size in sufficient quantity. The increased favour in which the diamond boring system is now held has caused a great demand for these tubes, and they are specially manufactured by an eminent Birmingham firm. The new pipes are required for the difficult process of enlarging and lining the bore-hole to the diameter considered recuisite before attempting to withdraw the broken rods, \&ce. Mr. Willett says:-

"The engineers have no doubt whatever of their ultimate success, and as the extraction of the rods is not a matter involving the expenditure of oux funds, we can only regret the loss of the long summer days, and take comfort from the assurance that, "after the enlargement and lining is accomplished, there is a much better prospect of obtaining the desired depth of 2,000 ft. than there was a year ago that we should reach half the distance $(1,000 \mathrm{ft}$.$) ,$ provided always that the requisite funds be forthcomizig:'"

He is anxious to dispel what he terms "the delusion" that no more money is required from the public in consequence of a Government grant to the work having been obtained. He states that the Chancellor of the Exchequer, with laudable foresight and prudence, has promised to assist on certain conditions, to do which-

"I. We must spend 400 . in boring tubes, \&c.

II. We must bore $100 \mathrm{ft}$, which will cost $200 \%$; and then, and not till then,

III. We can draw 100 . from the Exchequer, and so on, claiming $100 \%$. for every roo ft. actually explored."

The third and last year of the tenancy for carrying out the work has been entered on, and therefore the necessity of speedily resuming the operations is at once seen. The financial position is cheering, the present balance being 594l. $7 s, 9 \%$. The honorary secretary says :-

"We are greatly indebted to the Right Hon, the Chan- 